\title{
APLICAÇÃO DE MODELO DE AUTODEPURAÇÃO PARA AVALIAÇÃO DA QUALIDADE DA ÁGUA DO RIBEIRÃO DO OURO, ARARAQUARA-SP
}

\author{
COSTA, Daniel Jadyr Leite \\ Apoio técnico no Programa de Mestrado em Desenvolvimento Regional e Meio Ambiente do Centro \\ Universitário de Araraquara - Uniara, mestrando EESC/USP. \\ TEIXEIRA, Denilson \\ Professor-pesquisador do Programa de Mestrado em Desenvolvimento Regional e Meio Ambiente do \\ Centro Universitário de Araraquara - Uniara. Rua Carlos Gomes, 1338 - Centro - CEP 14801-340 - \\ Araraquara-SP. E-mail: dteixeira@uniara.com.br
}

\section{RESUMO}

Os modelos matemáticos utilizados para simular a qualidade e o processo de autodepuração de corpos d'água são importantes ferramentas de auxílio à gestão dos recursos hídricos. Assim, o objetivo principal deste trabalho foi aplicar um modelo matemático de qualidade de águas superficiais (QUAL-UFMG) para avaliar a qualidade das águas do ribeirão do Ouro, em Araraquara (SP). A partir dos perfis de oxigênio dissolvido (OD) e da demanda bioquímica de oxigênio $\left(\mathrm{DBO}_{5,20}\right)$, obtidos ao longo da seção longitudinal do rio, foi possível determinar as zonas de autodepuração e verificar o atendimento aos padrões de qualidade de águas estabelecidos na Resolução CONAMA 357/2005. O modelo e os resultados encontrados poderão subsidiar gestores e pesquisadores na prevenção, controle e estudos referentes à poluição desse corpo de água.

Palavras-chave: Modelagem Matemática; Qualidade das águas superficiais; Gestão de recursos hídricos; Modelo QUAL-UFMG.

\begin{abstract}
Mathematical models used to simulate the quality and the process of self-purification of water bodies are important tools to aid the management of water resources. The main objective of this paper was to apply a mathematical model (QUAL-UFMG) to evaluate the water quality of the stream "ribeirão do Ouro", Araraquara (SP). Based on the profiles of dissolved oxygen (DO) and of the biochemical oxygen demand $\left(\mathrm{BOD}_{5,20}\right)$ obtained along the longitudinal section of the river, it was possible to determine the areas of self-purification and to verify compliance with the standards of water quality established by Resolution CONAMA 357/2005. The model and the obtained results will be able to support managers and researchers in the prevention, control and studies related to pollution of this water body.
\end{abstract}

KEYwORDS: Mathematical Modeling; Surface water quality; Water resources management; Model QUALUFMG. 


\section{INTRODUÇÃO}

O atual modelo de desenvolvimento socioeconômico, o crescimento populacional acelerado e a necessidade de atendimento às diversas atividades humanas têm como resultado um panorama complexo em relação à degradação dos recursos naturais, especialmente evidenciado nos aspectos qualitativos e quantitativos dos recursos hídricos. Este cenário tornou ainda mais importante os estudos e procedimentos de gestão integrada desses recursos. Assim, cada vez mais, são necessárias ferramentas que quantifiquem direta ou indiretamente esses impactos ambientais, como passo inicial para a tomada de decisões que estejam direcionadas à manutenção e recuperação da qualidade desses sistemas.

Os modelos matemáticos utilizados para simular a qualidade da água e o processo de autodepuração de corpos d'água são importantes ferramentas de auxílio à gestão dos recursos hídricos, podendo atuar tanto na prevenção como no controle da degradação desses recursos.

Dentre as ações, subsidiadas por esses modelos, podem ser destacadas: a alocação de empreendimentos potencialmente geradores de poluição hídrica, a definição do nível de tratamento a ser empregado para que os padrões de qualidade não sejam infringidos, a delimitação de trechos críticos de poluição e a previsão das alterações da qualidade da água ao longo do tempo e do espaço em condições normais ou decorrentes de descargas acidentais. As duas últimas estão relacionadas com a determinação das zonas de autodepuração de um corpo hídrico.

A autodepuração pode ser definida como um processo natural de recuperação da concentração de saturação do oxigênio dissolvido (OD) no corpo de água, a jusante do recebimento de uma carga orgânica biodegradável. Para Von Sperling (2007), esse processo pode ser considerado como representativo de um fenômeno de sucessão ecológica, em que há uma sequência sistemática de substituições de uma comunidade aquática por outra, até que haja o equilibrio entre determinadas comunidades com as condições locais. Do início ao final desse processo estão presentes diferentes zonas de autodepuração.

O marco inicial do uso da modelagem nos estudos da poluição dos corpos hídricos foi em 1925, com o clássico modelo de Streeter-Phelps, que foi desenvolvido a partir de intensos estudos das fontes de poluição e seus impactos no rio Ohio, EUA (CHAPRA, 1997; THOMANN \& MUELLER, 1987; ORLOB, 1983). Este modelo descreveu o aumento, seguido de decréscimo, do déficit de oxigênio a jusante de uma fonte de material orgânico (GONÇALVES, 2009).

A inexistência de computadores limitava a aplicação do modelo analítico de Streeter-Phelps à cinética de primeira ordem, canais com geometria simples e escoamento em regime permanente e uniforme. Apenas no final da década de 1950, com o desenvolvimento dos métodos numéricos para solução de equações diferenciais com o uso de computadores, é que foi possível aumentar a complexidade dos modelos, sendo o Delaware Estuary Comprehensive Study Model (DECS), desenvolvido por Thomann em $1963^{1}$, o primeiro modelo de qualidade de águas baseado em expressões numéricas (CHAPRA, 1997).

O sucesso do modelo DECS encorajou os órgãos governamentais dos EUA a investirem na modelagem para o gerenciamento dos recursos hídricos; assim, outros modelos computacionais surgiram, os quais permitiram a simulação de mais variáveis de qualidade da água, de sistemas hídricos mais complexos - em regime permanente e não permanente - e maior flexibilidade nos ajustes dos coeficientes cinéticos (ORLOB, 1983).

Os modelos de qualidade de águas superficiais vêm sendo bastante utilizados, devido a sua credibilidade no meio técnico-científico, principalmente os modelos QUAL2K (uma versão atualizada e aprimorada dos modelos da família QUAL - I, II e 2E, este último distribuído pela USEPA $^{2}$ ) e o MIKE11, do grupo DHI software.

${ }^{1}$ THOMANN, R. V. Mathematical Model for Dissolved Oxygen. J. San. Eng. Div. ASCE89 (SA5): 1-30, 1963.

${ }^{2}$ United States Environmental Protection Agency. 
Vale salientar que, até o momento, a maioria dos trabalhos voltados para a modelagem de qualidade de águas superficiais foi realizada a partir de modelos unidimensionais, como Campos (1997); Gastaldni (2002); Kannel (2007); e Sardinha (2008). Como grande valia, principalmente para rios com vazões relativamente elevadas, está em ascensão a aplicação de modelos bi e tridimensionais, capazes de modelar a dispersão longitudinal e a difusão transversal de poluentes. As equações de conservação de massa e quantidade de movimento utilizadas nesses modelos podem ser resolvidas a partir de programas de fluidodinâmica computacional (ou computational fluid dynamics - CFD); essa abordagem está detalhada em Lien (1999); Ma (2002); Modenesi (2004); e Machado (2008).

O modelo utilizado neste trabalho foi o QUALUFMG (Von Sperling, 2007), que teve seu desenvolvimento baseado no QUAL2E. O QUALUFMG é um modelo unidimensional, de interface bastante amigável, adequado para rios com vazões relativamente baixas e escoamentos em regime permanente, pois essas condições não favorecem o fenômeno de dispersão longitudinal.

Esse modelo já foi usado nas simulações de diversos cenários para os cursos d’água componentes do Plano de Gerenciamento Integrado de Recursos Hídricos do
Distrito Federal (VON SPERLING, 2007).

Dessa forma, o objetivo principal deste trabalho foi justamente avaliar a qualidade das águas do Ribeirão do Ouro, em Araraquara (SP), a partir da aplicação de um modelo matemático de qualidade de águas superficiais (QUAL-UFMG).

A aplicação do modelo matemático produziu resultados numéricos da variação da concentração de Oxigênio Dissolvido (OD) e Demanda Bioquímica de Oxigênio $\left(\mathrm{DBO}_{5,20}\right)$ ao longo do rio. Os trechos críticos de degradação podem ser visualizados a partir do perfil de OD obtido, o qual forneceu subsídios para a verificação de atendimento à Resolução CONAMA 357/2005 e determinação das zonas de autodepuração do corpo hídrico. As zonas de autodepuração foram definidas e apresentadas em uma carta digital georeferenciada da bacia hidrográfica em estudo.

\section{Área de estudo: Bacia Hidrográfica do Ribeirão do Ouro}

A Bacia Hidrográfica do Ribeirão do Ouro pertence à Unidade de Gerenciamento de Recursos Hídricos Tietê-Jacaré (UGRHI-13) e localiza-se na porção central do Estado de São Paulo, no município de Araraquara (SP). Possui aproximadamente $84,3 \mathrm{~km}^{2}$ e abrange em torno de $45 \%$ da área urbana desse município (Figura 1). 

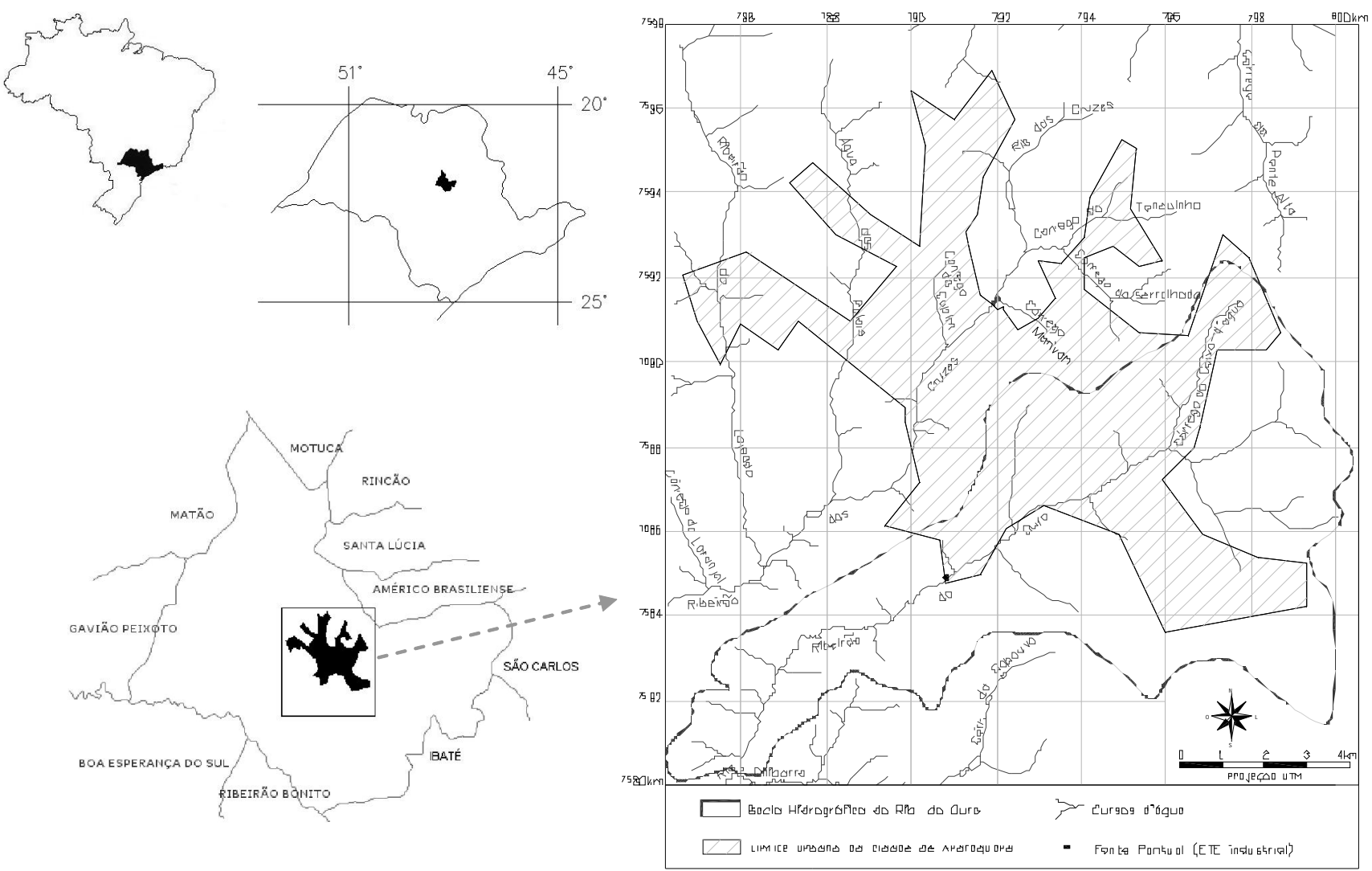

Figura 1 - Localização da Bacia Hidrográfica do Ribeirão do Ouro.

De acordo com Fuller (2008), pela classificação Koppen, essa bacia está localizada em uma região de clima "Tropical de Altitude" CWA, caracterizada por duas estações bem definidas: um verão com temperaturas altas (média de $31^{\circ} \mathrm{C}$ ) e pluviosidade elevada e um inverno com temperaturas amenas e pluviosidade reduzida.

Ummapeamento do uso e ocupação do solo, realizado a partir de imagens de satélite e apresentado por Teixeira et al. (2008), identificou que a Bacia Hidrográfica do Ribeirão do Ouro está ocupada predominantemente por áreas agrícolas e pastos, abrangendo uma área de 48,6 $\mathrm{km}^{2}$ (ou 57,6\% da área total da bacia). As áreas urbanas ocupam $27,1 \%$ e as áreas verdes (incluindo reservas legais e matas ciliares), apenas $13,3 \%$.

O Ribeirão do Ouro possui uma extensão aproximada de 19.900 metros. Ao longo de seu percurso recebe o lançamento de algumas fontes poluidoras; sendo assim, o modelo matemático utilizado neste trabalho foi aplicado para caracterizar o efeito desses lançamentos sobre a qualidade de suas águas. O trecho simulado inicia-se na seção transversal localizada a 13.400 metros de sua foz.

Na simulação realizada, verificou-se a presença de uma significativafonte pontual proveniente de umaindústria, e ainda, outras fontes pontuais lançadas direta e indiretamente no corpo de água. As fontes pontuais indiretas são advindas de tributários com elevadas concentrações de matéria orgânica. O Ribeirão do Ouro está enquadrado como Classe 4, de acordo com o Decreto 10.755, de 22 de novembro de 1977, que dispõe sobre o enquadramento dos corpos d'água receptores do Estado de São Paulo, conforme classificação prevista no Decreto 8.468, de 8 de setembro de 1976.

\section{Materiais e MÉTOdos}

Os dados de qualidade de águas de OD e DBO do Ribeirão do Ouro foram obtidos através de análises realizadas pelo Departamento Autônomo de Água e 
Esgotos (Daae), autarquia municipal. Os dados foram coletados nos pontos de amostragem apresentados na Figura 2, no dia 31 de maio de 2006, e utilizados como valores de referência para a calibração do modelo. As análises seguiram os padrões descritos na $20{ }^{a}$ edição do Standard Methods for Examination of Water and Wastewater (APHA, 1998).

As vazões do rio e de seus tributários foram calculadas através do método de regionalização hidrológica, por meio do Sistema de Informações para o Gerenciamento de Recursos Hídricos (SIGRH) do Estado de São Paulo. O valor de referência foi a descarga específica $\left[\mathrm{m}^{3} / \mathrm{s} . \mathrm{km}^{2}\right]$, obtida a partir da vazão média plurianual de uma série histórica de 34 anos de um posto fluviográfico do Daee, localizado na Fazenda
Palmeiras, Rio Chibarro, em Araraquara (SP). O cálculo da área da Bacia Hidrográfica do Ribeirão do Ouro foi realizado com uso do software Auto CAD, utilizando-se como base cartográfica a folha digitalizada SF-22-X-D-VI-4 do IBGE (1:50.000).

O percurso simulado tem uma extensão de 13.400 metros e foi dividido em cinco trechos, sendo eles (com relação à seção inicial): 1. 0-3100 m; 2. 3100$5200 \mathrm{~m} ; 3.5200-7200 \mathrm{~m} ; 4.7200-12800 \mathrm{~m}$; e 5. 12800-13400 m. Os critérios para a divisão de trechos estão baseados nas características hidráulicas do rio e na localização das fontes poluidoras, como é mostrado na Figura 2. Os trechos foram divididos em elementos computacionais de comprimento $(\Delta \mathrm{x})$ igual a 100 metros.

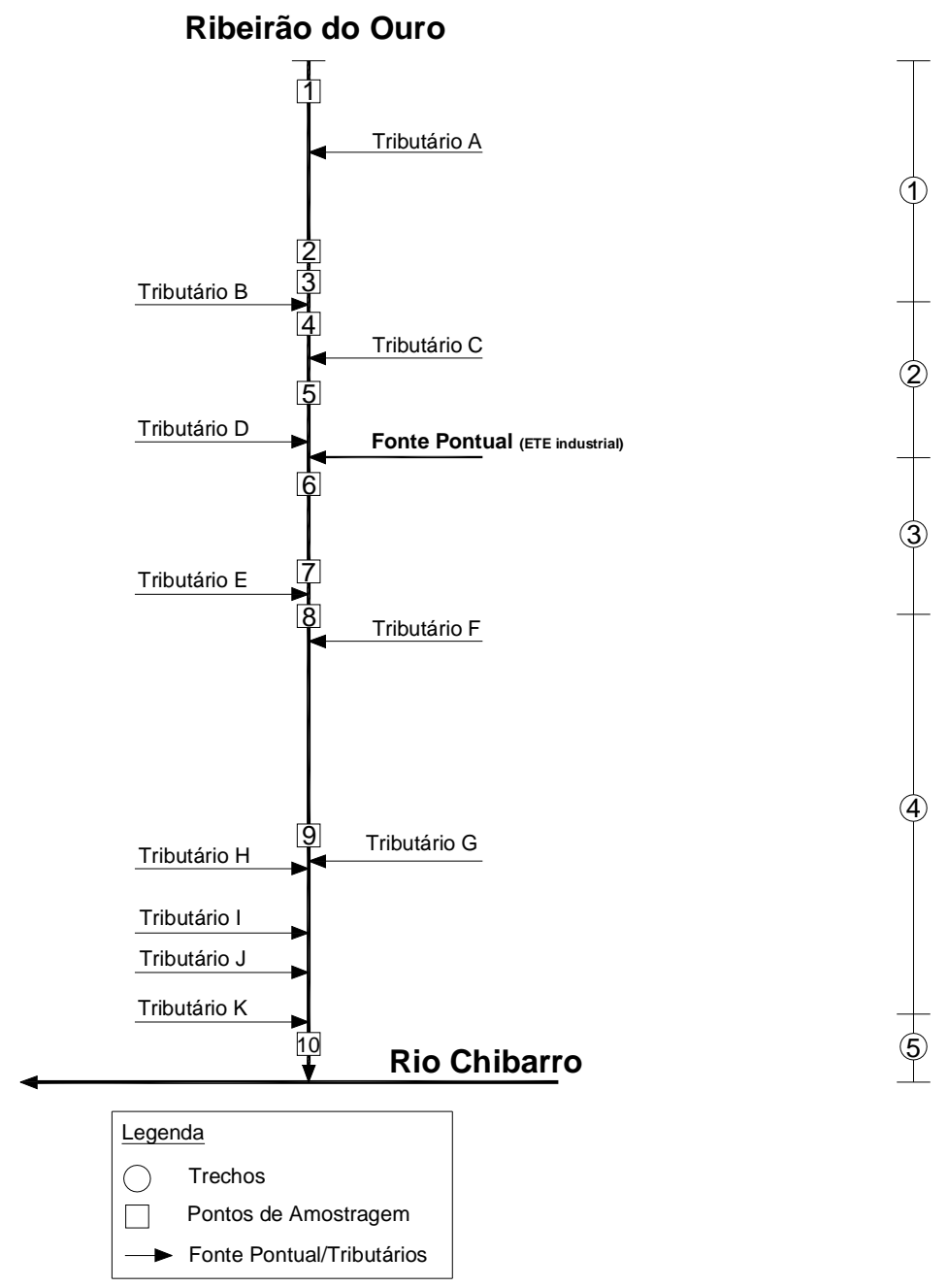

Figura 2 - Diagrama unifilar dos trechos simulados do Ribeirão do Ouro. 
Os dados de OD, DBO e vazão de lançamento pontual (industrial) foram estimados a partir de Ponezi (2000) e foram ajustados na etapa de calibração do modelo.

A declividade do leito do ribeirão foi determinada a partir da carta topográfica do IBGE (escala 1:50.000). A largura dos trechos foi medida e, a partir da vazão média, foram estimadas a profundidade e a velocidade média de cada trecho. A simulação foi realizada considerando o escoamento do rio sobre uma calha ideal, com geometria retangular. As características hidráulicas dos trechos simulados estão apresentadas na Tabela 1.

Tabela 1 - Características hidráulicas dos trechos simulados.

\begin{tabular}{c|c|c|c|c|c|c}
\hline Trecho & $\begin{array}{c}\text { Comprimento } \\
{[\mathbf{m}]}\end{array}$ & $\begin{array}{c}\text { Declividade } \\
{[\mathbf{m} / \mathbf{m}]}\end{array}$ & $\begin{array}{c}\text { Largura } \\
{[\mathbf{m}]}\end{array}$ & $\begin{array}{c}\text { Profundidade } \\
{[\mathbf{m}]}\end{array}$ & $\begin{array}{c}\text { Vazão }_{\mathbf{m}} \\
{\left[\mathbf{m}^{3} / \mathbf{s}\right]^{*}}\end{array}$ & $\begin{array}{c}\text { Velocidade }_{\mathbf{m}} \\
{[\mathbf{m} / \mathbf{s}]^{*}}\end{array}$ \\
\hline 1 & 3100 & 0,0065 & 3,6 & 0,4 & 0,334 & 0,23 \\
\hline $2^{* *}$ & 2100 & 0,0110 & 3,6 & 0,4 & 0,4876 & 0,34 \\
\hline $3^{* *}$ & 2000 & 0,0110 & 3,6 & 0,4 & 0,4876 & 0,34 \\
\hline 4 & 5600 & 0,0163 & 4,4 & 0,31 & 0,5743 & 0,42 \\
\hline 5 & 600 & 0,0057 & 5,3 & 0,55 & 0,6114 & 0,21 \\
\hline
\end{tabular}

*Valores médios para cada trecho;

**Os trechos 2 e 3 possuem características hidráulicas semelhantes: no entanto, essa divisão se deve ao lançamento pontual no início do trecho 3 , possuindo um coeficiente cinético de desoxigenação mais elevado, dado que a taxa de reação de degradação é proporcional à concentração de matéria-orgânica do meio (reação de primeira ordem).

Além dos dados de lançamento pontual da indústria, a calibração do modelo foi realizada pela variação dos coeficientes cinéticos $\left[\mathrm{T}^{-1}\right]$ de desoxigenação $\left(\mathrm{K}_{1}\right)$ e reoxigenação $\left(\mathrm{K}_{2}\right)$.

\section{Modelo matemático e solução das equações diferenciais ordinárias}

De uma maneira geral, as concentrações dos constituintes (como, por exemplo, o oxigênio dissolvido) em um corpo d'água são alteradas pelos mecanismos físicos de advecção-dispersão longitudinal e por processos bioquímicos e físicos de conversão.

De acordo com Von Sperling (2007) e Lima (1997), o transporte de poluentes em cursos d'água, em regime permanente, pode ser modelado desprezando-se o termo da dispersão longitudinal, visto que os gradientes de concentração são pequenos neste tipo de escoamento. Para o segundo autor, no caso de descargas acidentais concentradas, a dispersão deverá ser considerada, uma vez que se tem um grande gradiente de concentração.

A simulação realizada considerou o escoamento em regime permanente, desconsiderando assim a dispersão longitudinal. Por isso, para os cálculos dos perfis de concentrações de OD e DBO foi considerada a fluidodinâmica de um reator de fluxo em pistão, ou seja, apenas a advecção é utilizada como mecanismo de transporte.

As equações utilizadas em um modelo como este, para construção do perfil longitudinal de OD, são criadas a partir do balanço de massa desta variável, como mostrado na Figura 3. Apesar de não demonstrado, o mesmo balanço deve ser realizado para a DBO. 


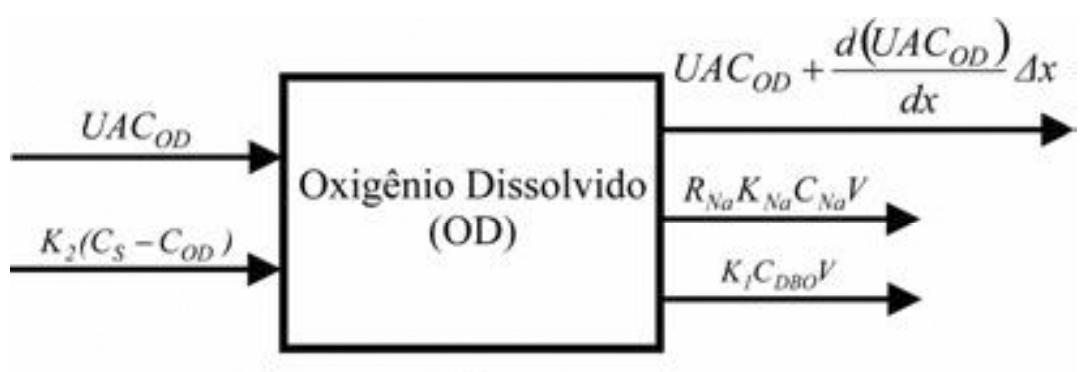

$\Delta \mathrm{x}$

Figura 3 - Balanço de massa para a variável OD.

O termo $\mathrm{R}_{\mathrm{Na}} \mathrm{K}_{\mathrm{Na}} \mathrm{C}_{\mathrm{Na}} \mathrm{V}$ se refere ao consumo de $\mathrm{OD}$, devido ao processo de nitrificação (DBO nitrogenada), o qual não foi considerado neste trabalho.

Aplicando-se o princípio de conservação da massa, obtém-se a Equação 1:

$V \Delta C_{O D}=\left[U A C_{O D}-\left(U A C_{O D}+U A \frac{\partial C_{O D}}{\partial x} \Delta x\right)+K_{2}\left(C_{S}-C_{O D}\right) V-K_{1} V C_{D B O}\right] \Delta t$

Dividindo os dois lados da equação por $\Delta \mathrm{t}, \mathrm{V}$ e considerando $\Delta \mathrm{xA}$ igual a $\mathrm{V}$, tem-se:

$\frac{\Delta C_{O D}}{\Delta t}=-U \frac{\partial C_{O D}}{\partial x}+K_{2}\left(C_{S}-C_{O D}\right)-K_{l} C_{D B O}$

Quando $\Delta \mathrm{t} 0$ :

$\frac{d C_{O D}}{d t}=-\frac{U \partial C_{O D}}{\partial x}+K_{2}\left(C_{S}-C_{O D}\right)-K_{1} C_{D B O}$

Considerando o regime permanente, tem-se:

$\frac{\partial C_{O D}}{\partial x}=\frac{K_{2}\left(C_{S}-C_{O D}\right)-K_{1} C_{D B O}}{U}$

emque:

$\Delta \mathrm{x}$ : comprimento do volume de controle [m];

$\Delta \mathrm{t}$ : intervalo de tempo [s];

A: área transversal do volume de controle $\left[\mathrm{m}^{2}\right]$;

$\mathrm{U}$ : velocidade média do escoamento $[\mathrm{m} / \mathrm{s}]$;

$\mathrm{C}_{\mathrm{OD}}$ : concentração de oxigênio dissolvido [mg/L];

$\mathrm{C}_{\mathrm{DBO}}$ : concentração de $\mathrm{DBO}[\mathrm{mg} / \mathrm{L}]$;

$\mathrm{C}_{\mathrm{s}}$ : concentração de saturação de oxigênio dissolvido [mg/L];

$\mathrm{K}_{1}$ : coeficiente cinético de desoxigenação $\left[\mathrm{dia}^{-1}\right]$; e

$\mathrm{K}_{2}$ : coeficiente cinético de reoxigenação [ $\left.\mathrm{dia}^{-1}\right]$. 
As equações diferenciais ordinárias apresentadas podem ser resolvidas a partir da aplicação de métodos numéricos. Vários métodos de integração numérica podem ser usados, o QUAL-UFMG emprega o de Euler; embora mais simples, requer passos de integração menores que os demais, como, por exemplo, o de Runge-Kutta de quarta ordem. Pela integração, segundo o método de Euler, tem-se a seguinte equação geral:

$C_{n+1}=C_{n}+($ taxa de reação $\times \Delta x)$ emque:

$\mathrm{Cn}+1$ : concentração em uma posição qualquer $\left[\mathrm{g} / \mathrm{m}^{3}\right]$; Cn: concentração na posição anterior $\left[\mathrm{g} / \mathrm{m}^{3}\right]$;

Taxa de reação: representa a declividade da reta usada para encontrar o próximo ponto; $\mathrm{e}$

$\Delta \mathrm{x}$ : passo de integração [m].

Na Tabela 2 é mostrado o resultado da aplicação do algoritmo de Euler às equações diferenciais, construídas a partir dos balanços de massa.

Tabela 2 - Solução numérica das equações diferenciais.

\begin{tabular}{|c|c|c|}
\hline Variável & Equação diferencial & Solução numérica \\
\hline $\mathrm{OD}$ & $\frac{\partial C_{O D}}{\partial x}=\frac{K_{2}\left(C_{S}-C_{O D}\right)-K_{l} C_{D B O}}{U}$ & $C_{O D n+1}=C_{O D n}+\left(\frac{K_{2}\left(C_{S}-C_{O D n}\right)}{U}-\frac{-K_{1} C_{D B O_{n}}}{U} \times \Delta x\right)$ \\
\hline $\mathrm{DBO}$ & $\frac{\partial C_{D B O}}{\partial x}=-\frac{K_{1} C_{D B O}}{U}$ & $C_{D B O n+1}=C_{D B O n}-\left(\frac{K_{l} C_{D B O n}}{U} \times \Delta x\right)$ \\
\hline
\end{tabular}

Verificação de atendimento à legislação e determinação das zonas de autodepuração

Para a verificação de atendimento à legislação ambiental, foi utilizado como referência os padrões de qualidade de águas presentes na Resolução CONAMA $357 / 2005$, para a classe em que o Ribeirão do Ouro se encontra enquadrado.

As zonas de autodepuração foram obtidas a partir do perfil de OD, construído com o uso do modelo, e foram caracterizadas utilizando-se as definições contidas em Von Sperling (2007).
A espacialização das zonas de autodepuração foi realizada integrando-se os dados numéricos do perfil de OD (calculados em uma planilha eletrônica do tipo Excel) na folha digitalizada e georeferenciada do IBGE (software AutoCAD).

\section{Resultados E Discussões}

Os valores de Oxigênio Dissolvido (OD) e Demanda Bioquímica de Oxigênio (DBO) do Ribeirão do Ouro utilizados no modelo encontramse na Tabela 3.

Tabela 3 - Valores de OD e DBO do Ribeirão do Ouro utilizados no modelo.

\begin{tabular}{l|c|c|c|c|c|c|c|c|c|c|c}
\hline \multirow{2}{*}{ Parâmetros } & \multicolumn{10}{c}{ Pontos de Amostragem } \\
\cline { 2 - 12 } & $\mathbf{1}$ & $\mathbf{2}$ & $\mathbf{3}$ & $\mathbf{4}$ & $\mathbf{5}$ & $\mathbf{6}$ & $\mathbf{7}$ & $\mathbf{8}$ & $\mathbf{9}$ & $\mathbf{1 0}$ & Padrão rio classe 4 \\
\hline OD $[\mathrm{mg} / \mathrm{L}]$ & 7,0 & 6,2 & 6,6 & 5,0 & 5,4 & 3,9 & 1,9 & 0,8 & 1,5 & 0,8 & superior a $2,0 \mathrm{mg} / \mathrm{L}$ \\
\hline DBO $[\mathrm{mg} / \mathrm{L}]$ & 0,8 & 1,9 & 6,6 & 17,4 & 12,2 & 6,6 & 110,2 & 97,5 & 82,0 & 75,3 & - \\
\hline
\end{tabular}


Com uma área de influência na bacia hidrográfica de $35,6 \mathrm{~km}^{2}$ e descarga específica de $0,00874 \mathrm{~m} 3 / \mathrm{s} \cdot \mathrm{km}^{2}$ (método de regionalização hidrológica), a vazão calculada para a seção inicial do trecho simulado foi de $0,311 \mathrm{~m}^{3} / \mathrm{s}$. Os valores das vazões e concentrações de OD e DBO, para os tributários e efluente industrial, estão apresentados na Tabela 4.

Tabela 4 - Vazões, OD e DBO dos tributários e efluente industrial.

\begin{tabular}{c|c|c|c|c|c|c|c|c|c|c|c|c}
\hline \multirow{2}{*}{ Parâmetros } & \multicolumn{10}{|c}{ Tributários } & \multirow{2}{*}{$\begin{array}{c}\text { Fonte Pontual } \\
\text { (ETE industrial) }\end{array}$} \\
\cline { 2 - 15 } & A & B & C & D & E & F & G & H & I & J & K & 0,1 \\
\hline Vazão $\left[\mathrm{m}^{3} / \mathrm{s}\right]$ & 0,037 & 0,043 & 0,058 & 0,004 & 0,004 & 0,008 & 0,01 & 0,008 & 0,006 & 0,01 & 0,013 & 5,0 \\
\hline OD $[\mathrm{mg} / \mathrm{L}]$ & 5,0 & 0,0 & 5,0 & 5,0 & 5,0 & 5,0 & 5,0 & 5,0 & 5,0 & 5,0 & 5,0 & 0,0 \\
\hline DBO $[\mathrm{mg} / \mathrm{L}]$ & 2,0 & 123,0 & 2,0 & 2,0 & 2,0 & 2,0 & 2,0 & 2,0 & 2,0 & 2,0 & 2,0 & 600,0 \\
\hline
\end{tabular}

A fonte pontual proveniente da indústria cítrica foi estimada com uma DBO de $1200 \mathrm{mg} / \mathrm{L}$. Devido à existência de uma estação de tratamento de efluentes, considerou-se para a simulação uma remoção de
DBO de 50\%. De acordo com o ajuste aos dados experimentais, os valores encontrados para os coeficientes cinéticos $\mathrm{K}_{1}$ e $\mathrm{K}_{2}$, nos diferentes trechos, estão apresentados na Tabela 5.

Tabela 5 - Valores dos coeficientes cinéticos utilizados para a calibração do modelo.

\begin{tabular}{c|c|c}
\hline Trecho & $\mathbf{K}_{\mathbf{1}} *\left[\mathbf{d}^{-\mathbf{1}}\right]$ & $\mathbf{K}_{\mathbf{2}} *\left[\mathbf{d}^{-\mathbf{1}}\right]$ \\
\hline 1 & 0,20 & 10,5 \\
\hline 2 & 0,20 & 10,5 \\
\hline 3 & 0,45 & 10,5 \\
\hline 4 & 0,40 & 11,0 \\
\hline 5 & 0,40 & 10,5 \\
\hline
\end{tabular}

* (base e, $20^{\circ} \mathrm{C}$ )

Cabe destacar, para efeito de comparação com outros modelos, que os coeficientes cinéticos estimados no processo de calibração do modelo utilizado nessa pesquisa se encontram na faixa de valores aceita pelo modelo QUAL2E, um dos mais utilizados no meio técnico-científico.

Os perfis de OD e DBO encontram-se nas Figuras 4 e 5 respectivamente. 


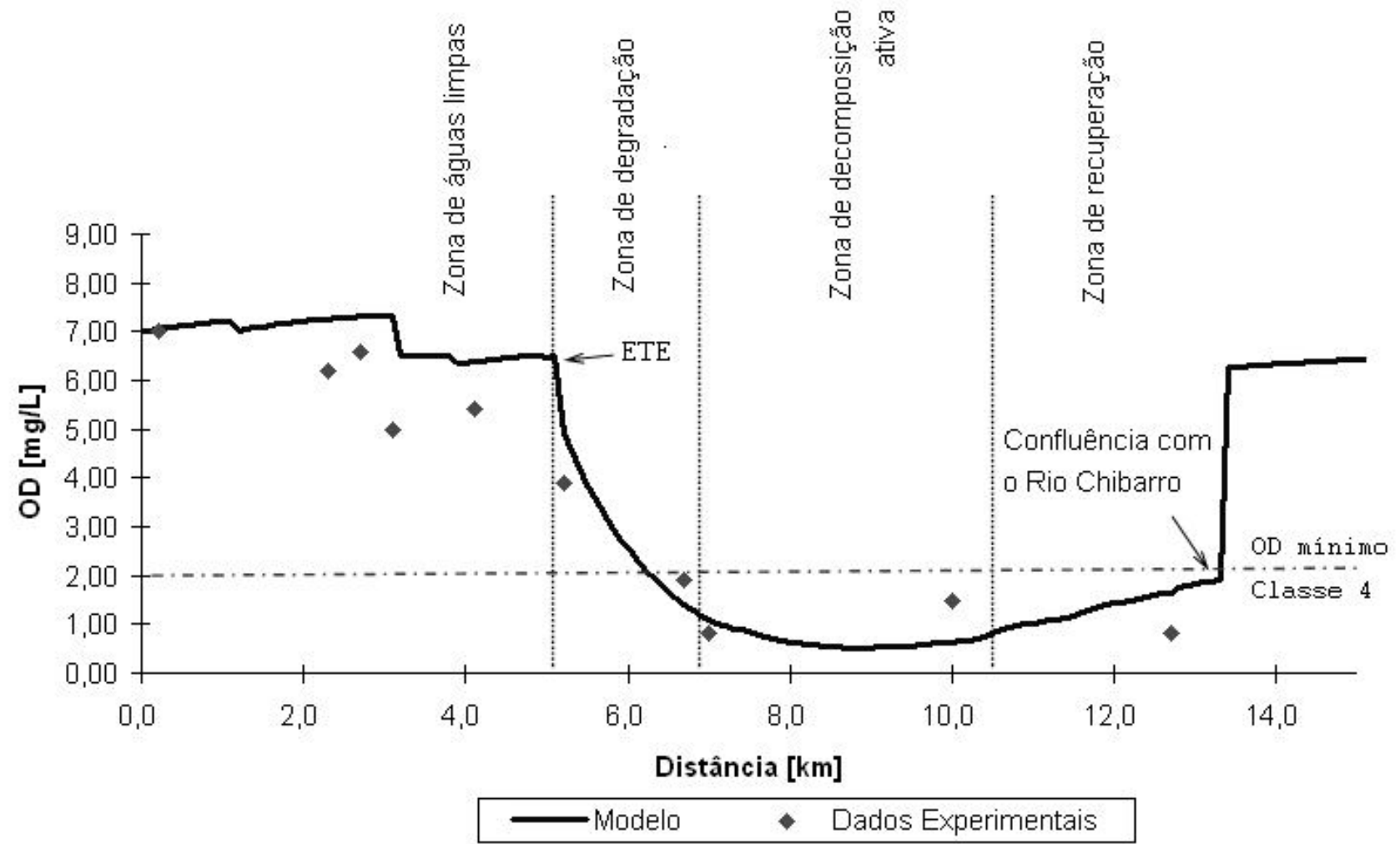

Figura 4 - Perfil de OD para os trechos simulados do Ribeirão do Ouro.

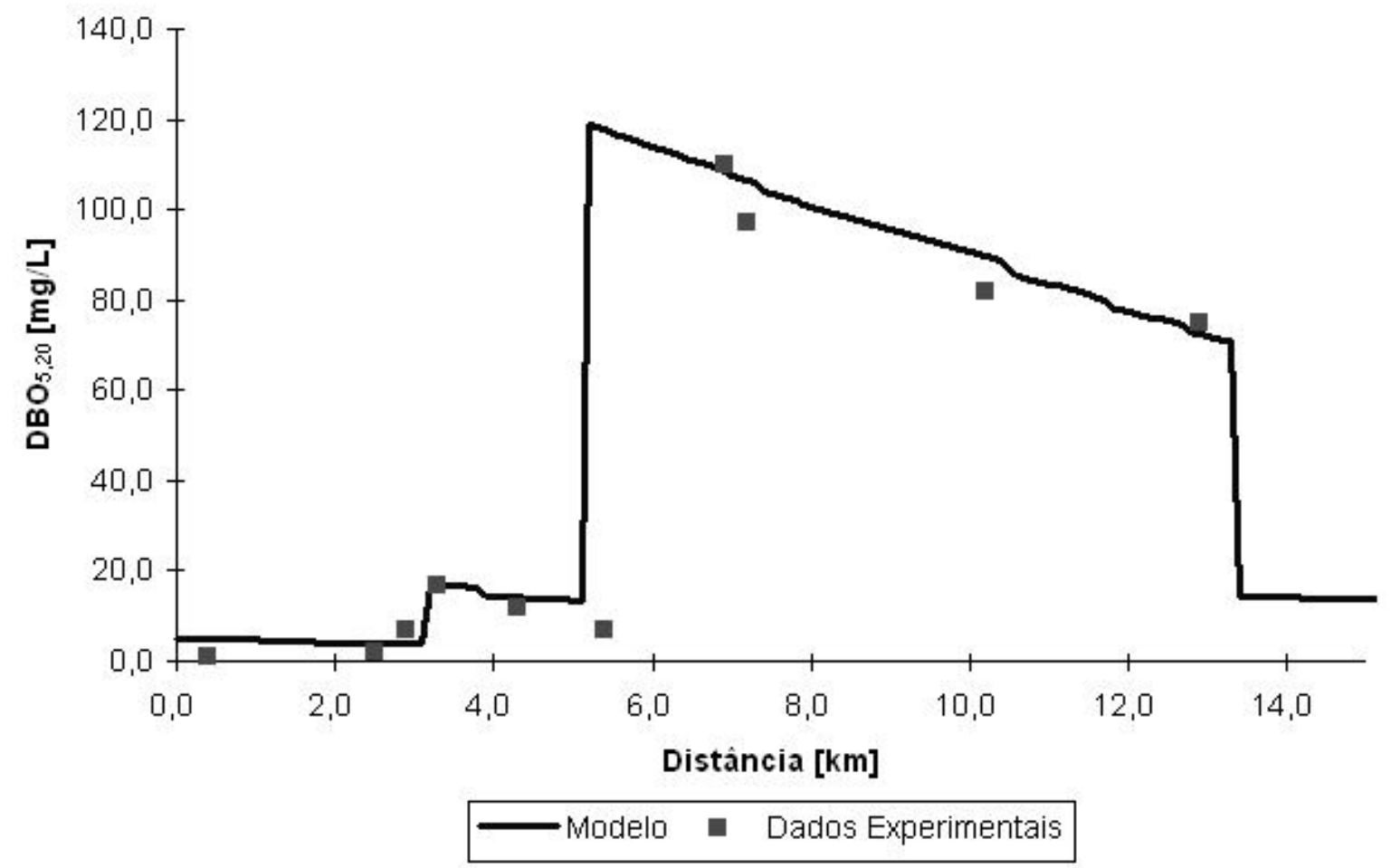

Figura 5 - Perfil de DBO para os trechos simulados do Ribeirão do Ouro. 
Nota-se nos resultados apresentados nas Figuras 4 e 5 significativa depleção da concentração de OD e aumento da DBO após o lançamento industrial. Embora menos significativo, a montante desse lançamento, o Tributário B (Figura 2) também contribui com o aporte de matéria orgânica para o sistema, aumentando a DBO e diminuindo os níveis de OD no corpo hídrico. Este tributário se encontra totalmente canalizado, escoando de forma subterrânea (abaixo de duas avenidas asfaltadas) sobre margens e leito de concreto. Devido a essas condições e aos resultados obtidos das análises físico-químicas da água, há prováveis lançamentos clandestinos no córrego.

A aplicação do modelo permite clara visualização dos trechos que se encontram dentro dos valores-limite estabelecidos por legislação. Verifica-se que na distância de 6,4 km (ou 6.400 metros de distância com relação à seção inicial do trecho simulado) até sua confluência como Rio Chibarro, as concentrações de OD se encontram inferiores a $2 \mathrm{mg} / \mathrm{L}$, ou seja, menores do que o padrão de qualidade determinado pela Resolução CONAMA 357/2005 para rios enquadrados na Classe 4 (Figura 4). O perfil de OD obtido também forneceu subsídios para a determinação e medição dos comprimentos longitudinais das zonas de autodepuração, que foram criadas a jusante do lançamento industrial.

Tomando-se como base a velocidade do escoamento em cada trecho, calculou-se o tempo de detenção hidráulica (TDH) das zonas de autodepuração. Esses valores podem ser visualizados na Tabela 6.

Tabela 6 - Zonas de autodepuração do evento simulado e respectivos tempos de detenção hidráulica.

\begin{tabular}{|c|c|c|}
\hline Zonas de Autodepuração & $\begin{array}{c}\text { Comprimento } \\
{[\mathrm{m}]}\end{array}$ & TDH* \\
\hline Águas Limpas & 5100 & $310,2 \mathrm{~min}=5 \mathrm{~h} 10 \mathrm{~min}$ \\
\hline Degradação & 1500 & $73,5 \mathrm{~min}=1 \mathrm{~h} 13 \mathrm{~min}$ \\
\hline Decomposição Ativa & 3700 & $151,5 \mathrm{~min}=2 \mathrm{~h} 31 \mathrm{~min}$ \\
\hline Recuperação & 6700 & $294,6 \mathrm{~min}=4 \mathrm{~h} 54 \mathrm{~min}$ \\
\hline
\end{tabular}

*Tempo de Detenção Hidráulica: é o tempo médio de permanência de uma partícula em suspensão em determinado trecho.

Após a fase inicial de perturbação do ecossistema (zona de degradação), os microrganismos desempenham ativamente suas funções de decomposição de matéria orgânica. Em consequência, o corpo d'água atinge seu nível mais acentuado de degradação, e a qualidade da água apresenta-se em seu estado mais deteriorado (VON SPERLING, 2007). Esses aspectos fornecem uma descrição qualitativa das condições ambientais predominantes no ecossistema aquático em uma zona de decomposição ativa. A correlação dessas condições com dados numéricos, permite inferir interpretações mais abrangentes sobre a perturbação causada no meio e suas possíveis consequências.

Considerando que as fontes pontuais presentes no Ribeirão do Ouro sejam lançadas em regime permanente, é possível, a partir dos dados da Tabela 6. verificar que haverá um trecho de 3.700 metros com TDH de aproximadamente duas horas e meia, governado por condições ambientais presentes na Zona de Decomposição Ativa.

A localização espacial das zonas de autodepuração da bacia hidrográfica do Ribeirão do Ouro para a simulação realizada está apresentada na Figura 6. 
A tradução desses resultados na forma de um mapa de zonas de autodepuração (qualidade das águas) constitui-se na mais completa forma de interação entre o pesquisador e os tomadores de decisão. Esse tipo de informação é muito importante para subsidiar o processo de gestão integrada dos recursos hídricos. Permite também, de interesse principalmente da academia, a definição de estudos e pesquisas voltadas aos seguintes temas: toxicologia, análise de risco, avaliação de impactos ambientais, indicadores biológicos de qualidade de água, entre outros. Cabe ainda destacar o potencial dessa ferramenta como subsídio para o estabelecimento de programas de monitoramento de qualidade de água para a sub-bacia hidrográfica em questão.

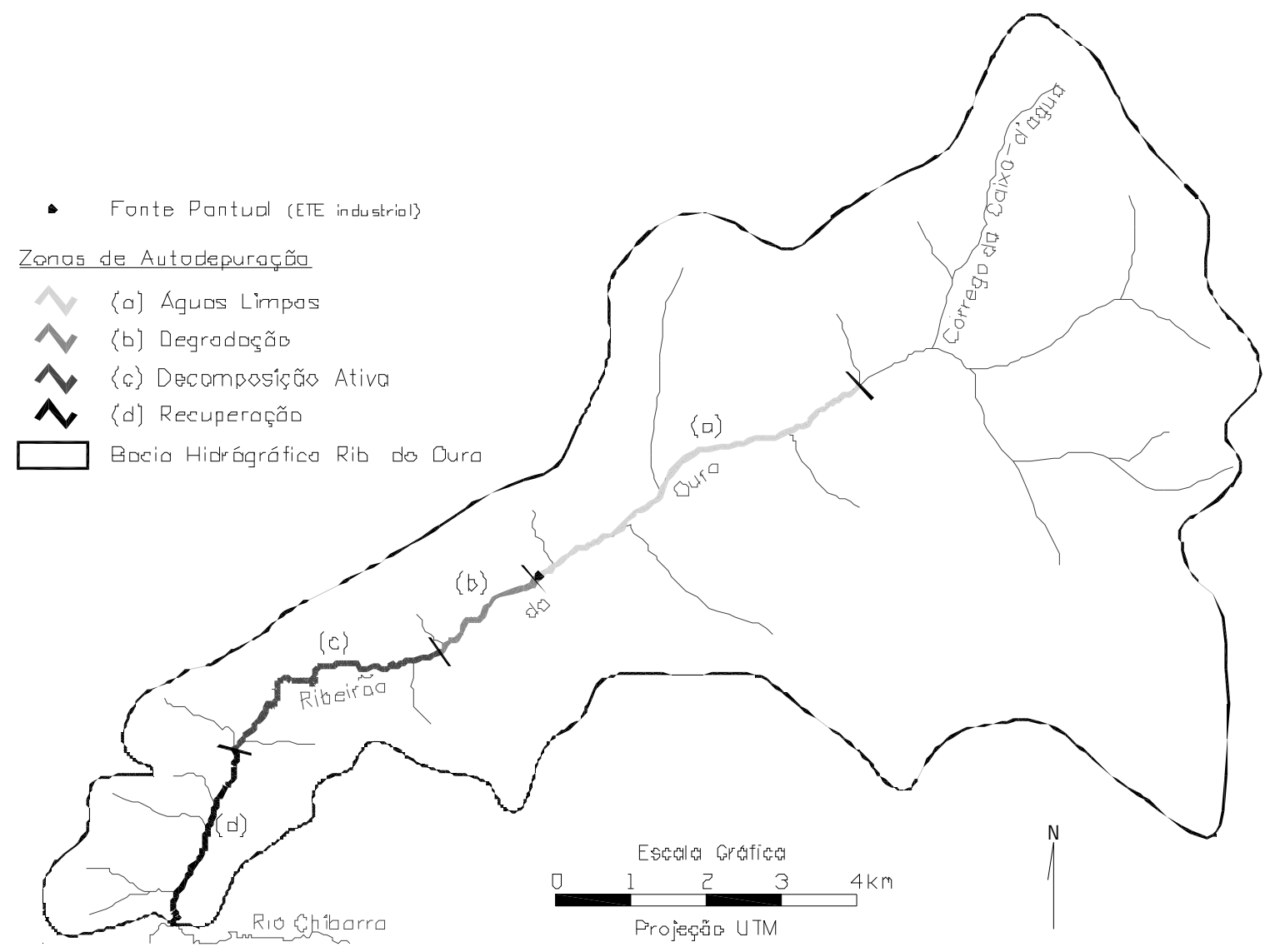

Figura 6 - Zonas de autodepuração da bacia hidrográfica do Ribeirão do Ouro para a simulação realizada.

\section{Conclusão}

Por meio das análises experimentais e do modelo, verifica-se que há um significativo impacto ambiental negativo no Ribeirão do Ouro, causado pelo lançamento de matéria orgânica proveniente principalmente de efluentes industriais.
O bom ajuste entre o modelo e os dados experimentais deve-se ao fato que as fontes pontuais e os coeficientes cinéticos de desoxigenação $\left(\mathrm{K}_{1}\right)$ e reoxigenação $\left(\mathrm{K}_{2}\right)$ foram utilizados como parâmetros de calibração.

O modelo poderá ser utilizado pelos gestores da 
bacia para subsidiar de forma preventiva e corretiva os problemas ambientais existentes no corpo de água.

Os estudos realizados apontam ainda para novas propostas de pesquisas, por exemplo, em função das incertezas dos valores de alguns dados utilizados no modelo como a vazão do rio, a vazão e concentração do efluente industrial e os coeficientes cinéticos $\mathrm{K} 1 \mathrm{e}$ $\mathrm{K} 2$, torna-se interessante a aplicação de métodos estatísticos que realizemuma simulação probabilística dos resultados obtidos. Esta simulação pode ser realizada através da Análise de Incerteza, por meio da Simulação de Monte Carlo.

\section{REFERÊNCIAS}

APHA. Standard Methods for the Examination of Water and Wastewater. American Public Health Association, 20 ${ }^{\text {th }}$ ed. Washington, 1998.

BRASIL (CONAMA). Resolução n. ${ }^{\circ} 357$ de 17 de março de 2005. Dispõe sobre as classificações dos corpo d' água e diretrizes ambientais para o seu enquadramento, bem como estabelece as condições e padrões de lançamento de efluentes, e dá outras providencias. 2005.

\section{CAMPOS, M. C. S. Utilização do modelo}

Qual2E para o estudo do decaimento bacteriano na Bacia dos Rio das Velha. Dissertação (Mestrado) - Universidade Federal de Minas Gerais, Belo Horizonte, 1997.

\section{CHAPRA, Steven C. Surface water quality modeling. New York, McGraw-Hill, 1997.}

\section{FULLER, Beatriz B. Caracterização espaço-} temporal dos recursos hídricos superficiais da sub-bacia do ribeirão do Ouro, Araraquara, SP. Dissertação (Mestrado) - Centro Universitário de Araraquara, Araraquara, SP. 157 p, 2008.

GASTALDINI, M. C. C. et al. Diagnóstico atual e previsão futura da qualidade das águas do Rio
Ibicuí utilizando o modelo QUAL2E. Engenharia Sanitária e Ambiental, vol. 7, n. 3 e n. 4, 2002.

GONÇALVES, J. C. S. I. Desenvolvimento de modelo numérico para a simulação da qualidade da água em rios utilizando o software Vensim PLE $^{\circledR}$. Dissertação (Mestrado) - Escola de Engenharia de São Carlos, Universidade de São Paulo, São Carlos. 203 p, 2009.

INSTITUTO BRASILEIRO DE GEOGRAFIA E ESTATÍSTICA. Carta do Brasil. Folha topográfica de Araraquara (SF-22-X-D-VI-4), São Paulo, IBGE. Escala 1:50.000, 1983.

KANNEL, P. R. et al. Application of QUAL2K for water quality modeling and dissolved oxygen control in the river Bagmati. Environ. Monit. Assess., vol.125, p.201-217, 2007.

LIEN, H.C et al. Bend-flow simulation using 2D depth-averaged model. Journal of Hydraulic Engineering, 125, Out. 1999.

LIMA, Carlos Antônio de A. O Uso do Modelo QUAL2E na Simulação da Qualidade das Águas do Rio Jacaré-Guaçu. Dissertação (Mestrado) Escola de Engenharia de São Carlos, Universidade de São Paulo, São Carlos. 179 p, 1997.

MA, L. et al. Computational fluid dynamics and the physical modeling of an upland urban river. Geomorphology, 44:375-391, 2002.

MACHADO, M. B. et al. Software para modelagem de dispersão de efluentes em rios. Engenharia Sanitária e Ambiental, vol. 13, n. 3, 2008.

MODENESI, K. et al. A CFD model for pollutant dispersion in rivers. Brazilian Journal of Chemical Engineering, v. 21, n. 4, p. 557-568, out./dez., 2004. 
ORLOB, G.T. Mathematical Modeling of Water Quality: Streams, Lakes, and Reservoirs. John Wiley \& Sons, 518p, 1983.

PONEZI, A. N. Tratamento de efluente líquido da indústria cítrica por lodo ativado por batelada (LAB): tratabilidade e microbiologia. Tese (Doutorado) - Universidade Estadual de Campinas, Faculdade de Engenharia Civil. Campinas, SP. 204 p, 2000.

SARDINHA, D. S. et al. Avaliação da qualidade da água e autodepuração do Ribeirão do Meio, Leme (SP). Engenharia Sanitária e Ambiental, vol. 13 , n. 3 e n. $4,2008$.

SÃO PAULO (Estado). Decreto n. ${ }^{\circ} \mathbf{8 . 4 6 8}$ de 8 de setembro de 1976. Aprova o Regulamento da Lei n. 997, de 31 de maio de 1976, que dispõe sobre a Prevenção e o Controle da Poluição do Meio Ambiente. 1976. Disponível em: <www.cetesb.sp.gov.br/Servicos/licenciamento/ postos/legislacao/Decreto_Estadual_8468_76.pdf > Acessado em: 02 de abril de 2010.

SÃO PAULO (Estado). Decreto n. ${ }^{\circ} \mathbf{1 0 . 7 5 5}$ de 22 de novembro de 1977. Dispõe sobre o enquadramento dos corpos de água receptores na classificação prevista no Decreto n. ${ }^{\circ} 8.468$, de 8 de setembro de 1976, e dá providências correlatas. 1977. Disponível em: <www.cetesb.sp.gov.br/ licenciamentoo/legislacao/estadual/decretos/ 1997_Dec_Est_10755.pdf >.Acessado em: 02 de abril de 2010.

\section{SISTEMA DE INFORMAÇÕES PARA O} GERENCIAMENTO DE RECURSOS HIIDRICOS. Regionalização Hidrológica do Estado de São Paulo. 2010. Disponível em < http:/ /www.sigrh.sp.gov.br/cgi-bin/regnet.exe?lig=podfp> Acessado em: 05 de maio de 2010.

TEIXEIRA, D. et al. Mapeamento do Uso e Ocupação do Solo e Análise de um Parâmetro Indicador de Qualidade da Água da Sub-bacia do Ribeirão do Ouro (Araraquara-SP). In: Anais do Fórum de Desenvolvimento Regional e Meio Ambiente Políticas, legislação e gestão ambiental, Araraquara-SP, 2008.

THOMANN, R. V.; MÜELLER, J.A. Principles of Surface Water Quality Modeling and Control. New York: Harper \& Row, Publishers, 644p, 1987.

VON SPERLING, M. Estudos e modelagem da qualidade da água de rios. Belo Horizonte:

Departamento de Engenharia Sanitária e Ambiental; Universidade Federal de Minas Gerais; 2007.

RECEBIDO EM 25/4/2010

ACEITO EM 15/7/2010 